\title{
Spinodal Decomposition in Thick InGaN Layers Studied by Strain Field Analysis
}

\author{
T. Bartel*, C. Kisielowski**, P. Specht*** \\ * Institut für Festkörperphysik, TU Berlin, Hardenbergstraße 36, 10623 Berlin, Germany \\ ** NCEM, Lawrence Berkeley National Laboratory, One Cyclotron Rd., Berkeley 94720, USA \\ *** Materials Science and Engineering, University of California, Berkeley 94720, USA
}

The ternary alloy InGaN has been subject to considerable investigation because of its importance as an active medium in electro optical devices. Early investigations [1] showed surprisingly that the InGaN thin films of these devices exhibit chemical fluctuation forming nanoscale Indium rich clusters. This may be a consequence of the wide miscibility gap of $\operatorname{In}_{\mathrm{x}} \mathrm{Ga}_{1-\mathrm{x}} \mathrm{N}$ alloys. However the driving force causing these fluctuations has yet to be established. Investigations of thicker InGaN layers promise to provide a more complete understanding of effects governing this phase separation, which is crucial to growing layers of better homogeneity. The main difference between thick layers and quantum wells is their strain state. The former are grown above the critical thickness and are in a partially relaxed state, while the latter are grown pseudomorphically and are strained by the substrate. Karpov performed calculations of the phase diagram of InGaN taking into account contributions of strain to the Gibbs free energy. It is shown that strain can effectively suppress decomposition for low $(\mathrm{x}<0.2)$ Indium concentration and the miscibility gap is skewed towards larger $\mathrm{x}$ [2]. However, it is still uncertain if decomposition in bulk and thin InGaN layers is governed by a similar process.

In previous studies both thin and thick InGaN layers with a wide range of Indium concentration showed a composition dependent amplitude of chemical fluctuations [3,4]. Other studies on thick InGaN layers reported periodic contrast fluctuations in TEM micrographs. Shoulders in diffraction spots were associated with these fluctuations and interpreted as chemical decomposition. The wavelength of the modulations is computed to be 20 to $3 \mathrm{~nm}$ for samples with Indium concentration between 0.21 and $0.34[5,6]$. Although these publications give convincing evidence of spinodal decomposition and ordering in InGaN thick layers, a quantitative study at a microscopic level is desirable.

In this talk we present the first quantitative measurement of periodic Indium concentration fluctuations in epitaxial $\operatorname{In}_{\mathrm{x}} \mathrm{Ga}_{1-\mathrm{x}} \mathrm{N}$ layers. This was achieved by strain field analysis from reconstructed high resolution TEM (HRTEM) micrographs to map local chemical composition. Strain mapping is a powerful tool which has been widely used to study InGaN quantum well heterostructures but there are no reports of the technique being applied to decomposed thick layers. Figure 1 a shows a HRTEM micrograph of a series of 20 images used for exit wave reconstruction. Strain mapping on the reconstructed image shows stripes of different Indium concentration (Fig. $1 \mathrm{~b}$ ) corresponding to contrast in the HRTEM image.

An interpretation of the fluctuations in the framework of the Cahn-Hilliard equations shows, that both wavelength and amplitude are important parameters for the understanding of spinodal decomposition in InGaN. Further we compare our results with previous studies on the amplitude of decomposition and suggest that very similar processes must govern phase separation in bulk and thin InGaN layers. 
References:

[1] C. Kisielowski et al., Jpn J. Appl. Phys. Vol 36 (1997), 6932

[2] S. Yu. Karpov, MRS Internet J. Nitride Semicond. Res. 3, 16 (1998)

[3] L.H. Robbins et al., IEEE Intl. Symp. Compound Semiconductors, October 2-5, 2000

[4] T. Bartel, Phys. stat. sol. (a) Vol 203 (2006), 167

[5]: $\quad$ A. N. Westmeyer et al., Appl. Phys. Lett. 79 (2001), 2710

[6]: $\quad$ M. Rao et al. Appl. Phys. Lett. 85 (2004), 1961

[7] The authors thank W. J. Schaff of Cornell University for the samples studied in this work. TB acknowledges financial support by the German National Academic Foundation.
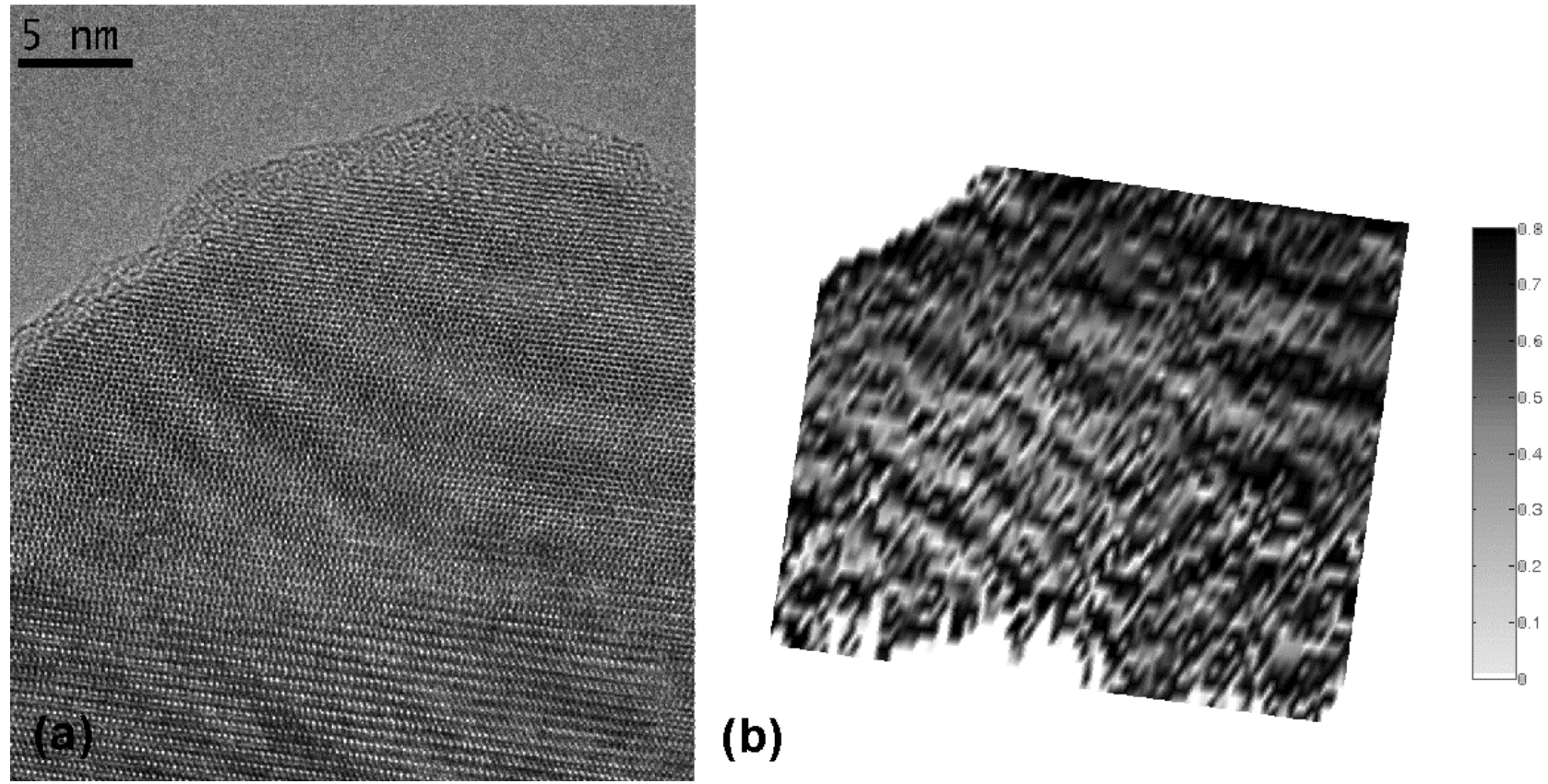

Fig. 1. (a) one of the HRTEM micrographs used for exit wave reonstruction. The InGaN layer shows contrast modulation corresponding to variations in c-lattice constant as extracted from the reconstructed image. (b) shows the strain map converted to Indium concentration (white areas correspond to vacuum or areas where no lattice constant could be extracted). 